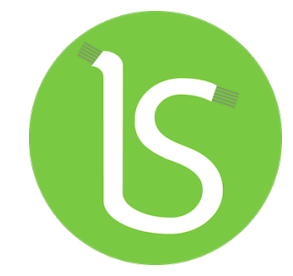

\title{
Revisión sistemática de la literatura sobre repositorios institucionales en México: importancia de la localización referencial de metadatos
}

\author{
Alma Delia Apale Zitzihua ${ }^{a}$ \\ Roberto Carlos Salas Castrob \\ Ignacio López Martínezc
}

Resumen - Esta investigación presenta un análisis sobre el estado particularmente de los artículos digitales de investigación almacenados en los Repositorios Institucionales de nuestro país, tomando una muestra de veinte de ellos incluido el Repositorio Nacional; se presenta como apoyo una concentración de datos importantes extraídos de la exploración Web que se llevó a cabo, además se fundamentó esta exploración realizando una Revisión Sistemática de la Literatura que contiene aportes importantes en el funcionamiento, desarrollo e implementación de estas plataformas en las instituciones de nivel superior, como resultado se presenta una propuesta de innovación para lograr un mayor impacto en la comunidad de usuarios así como una mejor manera de gestionar la productividad científica y académica resaltando la importancia de la referenciación de metadatos.

Palabras clave - Repositorio Institucional, Artículo, Acceso Abierto, Metadatos, Revisión Sistemática de la Literatura.

Abstract - This research presents an analysis on the state particularly of digital research articles stored in the Institutional Repositories of our country, taking a sample of twenty of them including the National Repository. It is presented as support a concentration of important data extracted from the Web exploration that was carried out, in addition this exploration was based on annexing a systematic review of the literature that contains important contributions in the operation, development and implementation of these platforms in higher level institutions, as a result it is presented a proposal for innovation to achieve a greater impact on the user community as well as a better way to manage scientific and academic productivity highlighting the importance of metadata referencing.

Keywords - Institutional Repository, Article, Open Access, Metadata, Systematic Literature Review.

\section{CÓMO CITAR}

HOW TO CITE:

Apale Zitzihua, A. D., Salas Castro, R. C., \& López Martínez, I. (2022). Revisión sistemática de la literatura sobre repositorios institucionales en México: importancia de la localización referencial de metadatos. Interconectando Saberes, (13), 47-57.

https://doi.org//0.25009/is.v0il3 .2727

Recibido: 29 de septiembre de 2021

Aceptado: 24 de enero de 2022 Publicado: 31 de enero de 2022

\footnotetext{
a Instituto Tecnológico de Orizaba, México. E-mail: almazitzihua@gmail.com

b Universidad Veracruzana, México. E-mail: rosalas@uv.mx

'Instituto Tecnológico de Orizaba, México. E-mail: ignacio.Im@orizaba.tecnm.mx
} 


\section{INTRODUCCIÓN}

Debido al gran avance de las tecnologías de la información (TIC) actualmente es más fácil difundir los productos resultantes de investigaciones en medios electrónicos, logrando un gran impacto y un mayor alcance. En particular, este trabajo aborda el estado actual de los artículos de divulgación que son almacenados en los Repositorios Institucionales (RI) de México mediante una exploración web realizada y con sustento básico en el libro Systematic Approaches to a Successful Literature Review (Booth et al., 2016).

El interés por los artículos como productos de investigación surgió debido a la digitalización actual de los artículos publicados y la posibilidad para encontrar puntos de oportunidad que lleven al desarrollo de nuevas herramientas tecnológicas de gestión que apoyen, complementen y reflejen la producción científica de las instituciones en conjunto con los RI.

De manera general se desglosa la adaptación de la metodología Revisión Sistemática de la Literatura, SLR (Booth et al., 2016, Xiao, Watson, 2019, González el al., 2020, Barrueco, Termens, 202I), que presenta en el inicio las preguntas a resolver en el desarrollo de la investigación, siguiendo con la definición del alcance en la cual se exponen los conceptos más relevantes, posteriormente la búsqueda y exploración, revisión de la literatura y selección de datos relevantes, finalmente se realizó la extracción de datos para escribir a partir de los resultados obtenidos.

\section{Metodología SLR}

El propósito principal de esta investigación fue conocer algunos aspectos importantes de los RI y el Open Access (OA) en nuestro país, este desarrollo se realizó con base en los siguientes cuestionamientos: ¿Cuál es el estado actual, en un sentido referencial, de los artículos de investigación dentro de los RI en nuestro país?, ¿Cuáles son los principales $R I$ y desde qué instituciones son operados?, y finalmente, ¿Cuántos de ellos cuentan con la opción de búsqueda de artículos?

Por lo tanto, fue necesario llevar a cabo una revisión exhaustiva y sistemática de la literatura referente a la temática que gira en torno a RI, para esto se utilizó la metodología SLR.

\section{Figura I}

Proceso de la metodología Revisión Sistemática de la Literatura modificada

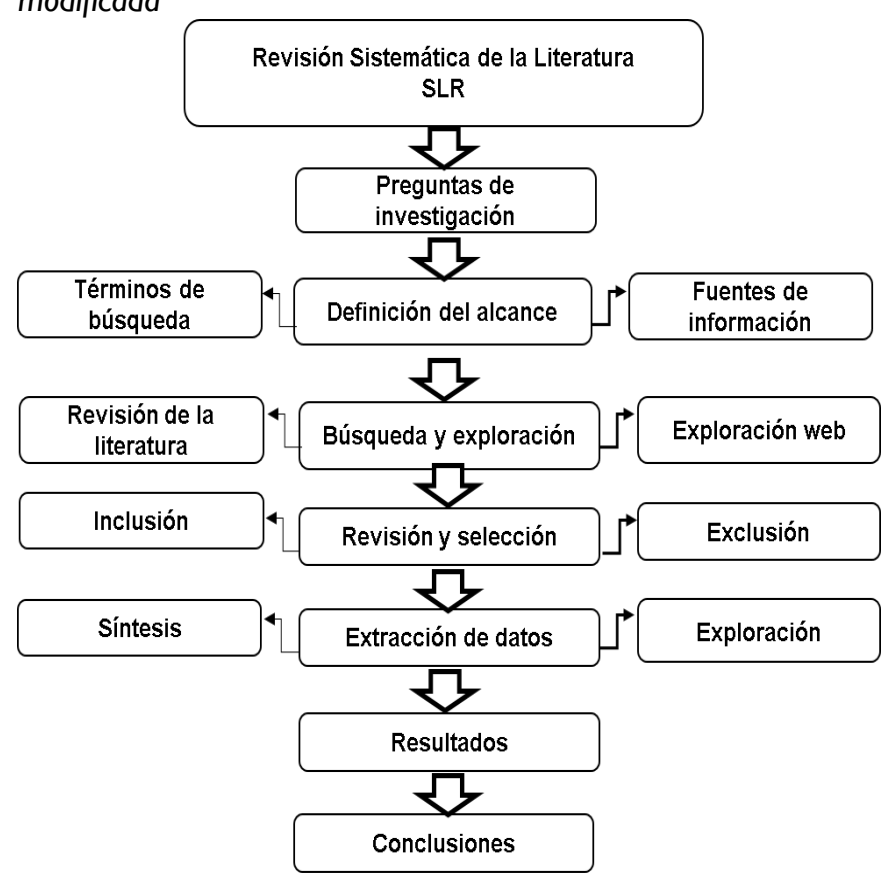

Nota: Obtenido de Proceso adaptado de la SLR de Booth (2016).

En consecuencia, se llevó a cabo una adaptación del proceso SLR específicamente para esta investigación, Figura I. 


\section{DEFINICIÓN DEL ALCANCE}

En primer lugar, fue indispensable definir los principales términos a abordar en esta investigación, y se presentan a continuación:

- Repositorio Institucional: es una plataforma digital en la que se acumulan, administran y difunden los productos resultantes de investigaciones de los miembros de las instituciones científicas o académicas. Un dato relevante que se estipula en el documento Lineamientos Generales ubicado en el Repositorio Nacional dice que las investigaciones financiadas con recursos públicos deben estar disponibles al público en general, además se resalta la importancia de dar a conocer los avances de las investigaciones para mostrar el impacto de la producción científica a nivel nacional e internacional, la difusión tiene como resultado el logro de la retroalimentación por más expertos en el tema, así como facilitar el acceso a esta información a quienes les sea útil para derivar otra investigación. Debido a esto el contenido de esta plataforma digital debe cumplir requisitos como ser una obra de calidad, actualizarse constantemente, seguro y por supuesto cumplir con los aspectos legales como el respeto a los derechos de propiedad intelectual (CONACYT, 202I; Cossio et al., 2017).

- Evaluación por pares: de acuerdo con las definiciones expuestas en el capítulo tercero del documento Lineamientos específicos para repositorios (LER) Capítulo I Disposiciones Generales, la evaluación por pares es un proceso que tiene como objetivo validar la calidad, originalidad, factibilidad, rigor científico y demás aspectos de importancia de los productos que serán almacenados en el RI (CONACYT, 202I).

- Metadatos: son un conjunto de elementos de información estructurados, que empezaron a utilizarse por primera vez en 1968 en el ámbito informático, sirven para vincular un objeto digital, en este contexto se utilizan principalmente los de tipo descriptivos y son utilizados por una amplia variedad de bases de datos como un medio de identificación y organización muy importante al momento de gestionar las investigaciones (RamosSimón, Cobo-Serrano, 2018).

- Acceso Abierto: el OA (Open Access por sus siglas en inglés) es una iniciativa que tiene como objetivo brindar acceso en línea gratuito a la información y al uso sin restricciones de los recursos digitales siempre y cuando se cumplan ciertas condiciones como: sin importar el medio el contenido cuente con acceso libre y universal tomando en cuenta los derechos de autor al publicar el contenido de su obra para obtener sus respectivos créditos, además de que la publicación se debe realizar con un formato apropiado (UNESCO, 202I). En la guía para la evaluación de repositorios institucionales de investigación se exponen las siguientes ventajas del OA:

La investigación se hace más efectiva y sus resultados más visibles cuando se eliminan las barreras legales, comerciales y tecnológicas de acceso a la información científica. Además, el acceso abierto previene la duplicación, fomenta la transferencia tecnológica y de conocimiento y promueve la innovación. Todos los actores implicados en el sistema de comunicación de la información científica se benefician del acceso 
abierto a la investigación y a los datos generados por ésta. (Cruz et al., 2017, p. 4)

- Artículo: es el medio por el que se difunde un trabajo de investigación que tiene como finalidad dar a conocer los resultados obtenidos en el ámbito académico o científico, para esto debe cumplir con requisitos como presentar datos válidos y fidedignos, estar escrito en un lenguaje claro, preciso y que utilice una estructura normalizada. Es requisito para publicarse en una revista indexada o almacenarse en el RI haber pasado por un proceso de evaluación por expertos (mejor conocida como evaluación por pares) que garantice la pertinencia y el valor de su aportación para llevar a cabo la difusión de este (Booth et al., 2016, Hernando, 2019).

- Revisión sistemática de la literatura: Booth et al. (2016) define la revisión sistemática como una pregunta que es formulada claramente, que utiliza métodos sistemáticos y explícitos para identificar, comparar, seleccionar y valorar críticamente una investigación de gran importancia en la que se recogen datos cualitativos $y$ cuantitativos de la literatura existente que finalmente se analizan y se obtienen resultados que responden dicho cuestionamiento. La delimitación de estos conceptos relevantes para la presente revisión fue importante para encaminar las preguntas planteadas de esta investigación, enfocadas principalmente a los RI de nivel superior. Con base en ellas, la búsqueda se centró en artículos ubicados en las siguientes bases de datos: SCIENCEDIRECT, EBSCO, REDALYC.

De la misma forma se llevó a cabo la exploración web de una muestra de veinte repositorios de las principales instituciones de educación superior IES de nuestro país para evaluar el estado actual de su funcionamiento.

\section{BÚSQUEDA Y EXPLORACIÓN}

En esta fase se realizó la exploración web, así como la búsqueda y recolección de la literatura en las bases de datos antes mencionadas utilizando la palabra clave principal de esta investigación: Repositorio Institucional (ver Figura 2).

\section{Figura 2}

Resultados de la búsqueda de artículos en las bases de datos

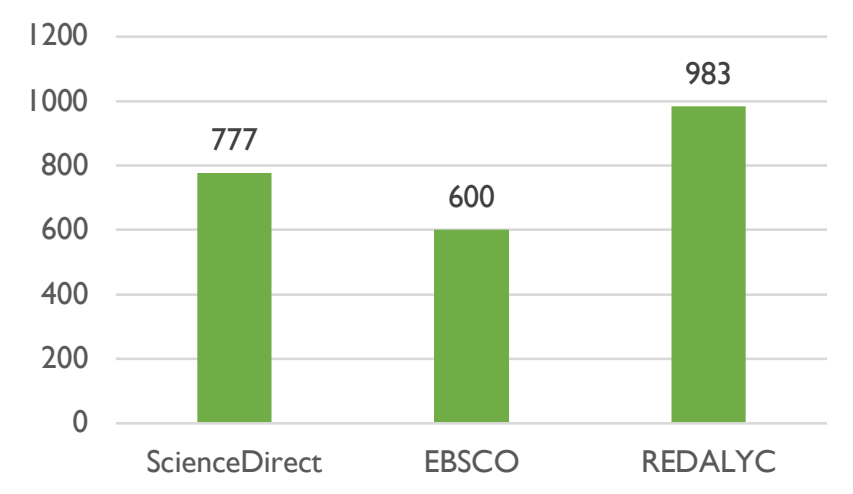

Los resultados mostrados corresponden a la búsqueda general aun sin aplicar los filtros para una selección apropiada de la literatura.

\section{REPOSITORIOS DE INSTITUCIONES DE EDUCACIÓN SUPERIOR EXPLORADOS}

En la Tabla I se presenta el listado en orden alfabético de la muestra de las IES en las cuales se realizó la exploración de repositorios institucionales, en su mayoría aquellas instituciones de México que son financiadas con fondos públicos, incluyendo el Repositorio Nacional. En esta información se destaca el objetivo que cada institución presenta al ingresar a su sitio web. 


\section{Tabla I}

Listado de RI explorados pertenecientes a IES de México

RI

\begin{tabular}{l} 
BUAP \\
Benemérita Universidad \\
Autónoma de Puebla \\
\hline CETYS UNIV. \\
Centro de Enseñanza Técnica \\
y Superior \\
COLMEX \\
Colegio de México \\
\hline
\end{tabular}

IPN

Instituto Politécnico Nacional

ITESM

Instituto Tecnológico y de

Estudios Superiores de

Monterrey

ITO

Instituto Tecnológico de

Orizaba

TecNM

Tecnológico Nacional de

México

UACJ

Universidad Autónoma de

Ciudad Juárez

UAM

Universidad Autónoma

Metropolitana

UAN

Universidad Autónoma de Nayarit

\section{UANL}

Universidad Autónoma de

Nuevo León

UASLP

Autónoma de San Luis Potosí

UdeG

Universidad de

Guadalajara

UDLAP

Universidad de las Américas

Puebla

UIA PUEBLA

Universidad Iberoamericana

UNAM

Universidad Nacional

Autónoma de México

UPN

Universidad Pedagógica

Nacional

UTCV

Universidad Tecnológica del

Centro de Veracruz

\section{Objetivo}

En este repositorio institucional de acceso abierto los docentes e investigadores de la BUAP, depositan sus documentos en acceso abierto.

No presenta

No presenta

Esta plataforma tiene como objetivo almacenar, preservar y difundir la producción científica y académica de la comunidad politécnica en formato digital. Así como maximizar la visibilidad, el uso y el impacto de la producción científica y académica de la comunidad Politécnica

El Repositorio Institucional del Tecnológico de Monterrey (RITEC) preserva, organiza y garantiza la visibilidad y acceso a la producción administrativa, académica y científica de la institución, así como al patrimonio documental y artístico que se encuentra bajo su resguardo y que está disponible en Acceso Abierto.

No presenta

Bienvenido al Repositorio Institucional de Acceso Abierto del Tecnológico Nacional de México, que almacena y organiza la documentación y producción de índole científica y académica con el propósito de preservarla en formato digital y facilitar su acceso y visibilidad global.

No presenta

En este Repositorio los docentes e investigadores de la UAM Azcapotzalco, depositan sus documentos en acceso abierto.

La Universidad Autónoma de Nayarit convencida y comprometida con la Estrategia Nacional implementada por el CONACYT para brindar acceso abierto a la información científica, tecnológica y de innovación generada con fondos públicos, generó este Repositorio con el objetivo de que los resultados de la investigación, el desarrollo tecnológico, la generación de nuevos productos, procesos y servicios derivados de la innovación, así como los proyectos finales de posgrado, se difundan a nivel local, nacional e internacionalmente.

No presenta

Bienvenido al Repositorio Institucional de Acceso Abierto de la UASLP que almacena y organiza la documentación y producción universitaria de índole científica, académica y administrativa, con el propósito de preservarla en formato digital y facilitar su acceso y visibilidad global.

El Repositorio Institucional de la Universidad de Guadalajara facilita el acceso virtual, libre y abierto al conocimiento académico y científico producido en la Universidad de Guadalajara para incrementar las posibilidades de satisfacer las necesidades informativas de nuestra comunidad, así como ampliar su visibilidad internacional, uso e impacto.

Bienvenido al Repositorio Institucional de la Universidad de las Américas Puebla RI-UDLAP un proyecto financiado por CONACYT para interactuar con el Repositorio Nacional.

En este repositorio institucional de la Universidad Iberoamericana de Puebla contiene archivos electrónicos en formato de publicaciones editadas por la universidad que han sido distribuidas anteriormente en su versión impresa sobre papel o bien que se hacen públicos por primera vez aquí. Estos archivos se producen de manera íntegra con propósitos de difusión y divulgación académica, y por ende no poseen fines de lucro.

El Repositorio Institucional de la UNAM permite integrar, publicar y consultar los contenidos académicos que genera y resguarda la Universidad.

Bienvenido al Repositorio Institucional de la Universidad Pedagógica Nacional. Esta herramienta recoge, conserva y distribuye la producción digital de carácter científico, académico y cultural de la Institución, con el propósito fundamental de difundir conocimiento relacionado con la educación y la pedagogía.

El Repositorio Institucional "REInl" de acceso abierto, permite facilitar el acceso a los Recursos de Información Académica, Científica, Tecnológica y de Innovación (RIACTIs) de la Universidad Tecnológica del Centro de Veracruz, Además es una fuente de divulgación científica y tecnológica que cumple y atiende los lineamientos generales del Repositorio Nacional, respetando los derechos de autor vigentes. 


\begin{tabular}{ll}
\hline $\begin{array}{l}\text { UV } \\
\text { Universidad Veracruzana }\end{array}$ & No presenta \\
\hline $\begin{array}{l}\text { Repositorio Nacional } \\
\text { (CONACYT) }\end{array}$ & $\begin{array}{l}\text { El Repositorio Nacional es una plataforma digital que proporciona acceso abierto en texto completo a } \\
\text { diversos recursos de información académica, científica y tecnológica, es decir, sin requerimientos de } \\
\text { suscripción, registro o pago. En el Repositorio pueden ser consultados, entre otros materiales: artículos de } \\
\text { Consejo Nacional de Ciencia } \\
\text { y Tecnología }\end{array}$ \\
$\begin{array}{l}\text { memorias de congresos y patentes, así como otros documentos académicos que se producen en México con } \\
\text { fondos públicos. }\end{array}$
\end{tabular}

En esta acción se analizó el estado de cada RI, así como los tipos de productos que almacena y gestiona, pero con principal atención en las opciones relacionadas con artículos, debido al impacto e importancia que estos tienen en el proceso de desarrollo y conclusión de una investigación. Es importante resaltar que el Repositorio Nacional se rige bajo ciertos lineamientos generales y legales que se exponen dentro del mismo, para operar en conjunto con las instituciones como una forma de gestionar que el contenido sea el adecuado y cumpla con los requerimientos de publicación.

\section{ASPECTOS LEGALES Y LINEAMIENTOS GENERALES DEL REPOSITORIO NACIONAL}

En el sitio web del Repositorio Nacional en el apartado de Colaboración se explican los requisitos que se deben cumplir para publicar contenido (artículos, tesis, libros, entre otros) en los RI. A continuación, se enlistan los más relevantes:

- Para colaborar es necesario ser tecnólogo, académico o estudiante en algún nivel superior.

- Entre los tipos de trabajos a depositar se encuentran: artículos, tesis, ponencias, prototipos, entre otros.

- Los tipos de publicaciones deben ser resultado de investigación científica y tecnológica.
- Es indispensable que todo trabajo a depositar debió ser revisado por pares y contar con el permiso de publicación por parte del autor para publicarse como OA.

- Es necesario contar con una licencia de contenidos abiertos o contar con un documento de periodo de embargo

Todo lo anterior se estipula en la documentación concentrada en el Repositorio Nacional (CONACYT, 202I).

\section{REVISIÓN Y SELECCIÓN DE LA LITERATURA}

Para la selección de la literatura fue necesario aplicar filtros de búsqueda correspondientes, a continuación, se muestran los datos de inclusión y exclusión utilizados en esta revisión:

\section{Tabla 2}

Inclusión y exclusión de datos en la búsqueda en las bases de datos

\begin{tabular}{|c|c|c|}
\hline Datos & Inclusión & Exclusión \\
\hline Tipo de artículo & Investigación & Opinión \\
\hline Palabra clave & $\begin{array}{l}\text { Repositorio } \\
\text { Institucional }\end{array}$ & Instituciones \\
\hline Temporalidad & $2017-2021$ & $\begin{array}{c}\text { Años anteriores } \\
\text { a } 2017\end{array}$ \\
\hline Área temática & $\begin{array}{l}\text { Ciencias de la } \\
\text { computación }\end{array}$ & Ciencias Sociales \\
\hline Temas & $\begin{array}{l}\text { Software } \\
\text { Educación } \\
\text { Computación }\end{array}$ & Medicina \\
\hline Tipo de acceso & $\begin{array}{l}\text { Computacion } \\
\text { Acceso abierto }\end{array}$ & Archivo abierto \\
\hline
\end{tabular}


En esta fase se aplicaron los filtros presentados en la tabla y se describen a continuación:

- Tipo: se incluyeron sólo los artículos de investigación concentrados en revistas como: "Revista de sistemas y software", "Revista de bibliotecología académica", "Información, cultura y sociedad: revista del Instituto de Investigaciones Bibliotecológicas", "Profesional de la información", "Revista Internacional de Filosofia Iberoamericana y Teoría Social", entre otros, referentes a la temática desarrollo de software y educación. Se excluyeron aquellas revistas de ciencias sociales y medicina.

- Palabras clave: de forma general en las bases de datos se realizó la búsqueda utilizando la palabra principal de esta investigación Repositorio Institucional, ya que, con la información recabada se respondió el estado actual de los RI en nuestro país.

- Temporalidad: se incluyeron sólo los artículos de investigación publicados en el rango 2017-2021 y se descartaron aquellos que no estaban dentro de este rango.

- Área temática: Respecto al área temática se excluyeron aquellos artículos que aparecieron en la búsqueda enfocados en ciencias sociales y de la salud debido al tema de investigación planteado.

- Tipo de acceso: como último filtro se seleccionaron solo los documentos de acceso libre de las tres bases de datos elegidas, descartando los archivos de acceso libre temporal o con periodo de embargo.

\section{Figura 3}

Resultados de la fase de revisión y selección de la literatura

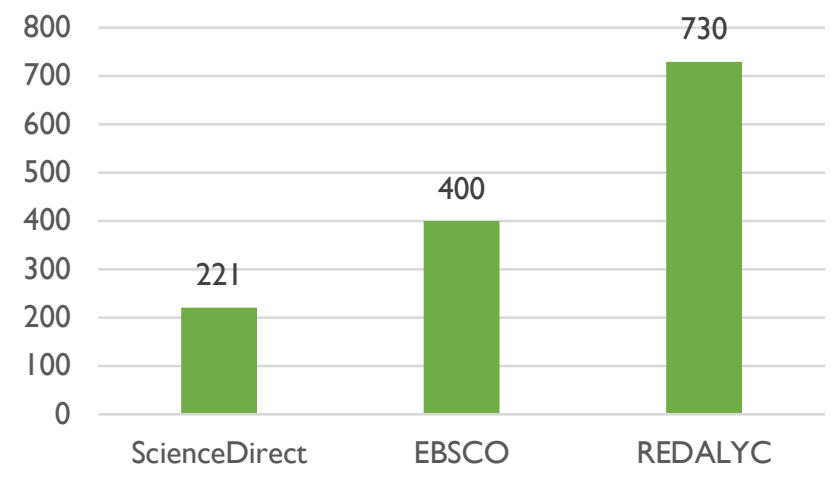

En resumen, los datos reflejados en esta última gráfica (Figura 3) son el resultado de la búsqueda deseada aplicando los filtros antes descritos para facilitar la localización de datos importantes y relevantes que apoyen el sustento de esta investigación.

\section{EXTRACCIÓN DE DATOS}

En esta fase del proceso se realizó la extracción de datos importantes respecto al tema en cuestión. La revisión sistemática sirvió para contrastar investigaciones, comparar opiniones, observar métodos y metodologías distintas utilizadas actualmente por las instituciones de educación para la preservación de su productividad científica y tecnológica.

A continuación, se presenta la literatura seleccionada sobre el resultado de investigaciones similares.

Este trabajo está enfocado principalmente al artículo de investigación que forma parte de los productos académicos resultantes de investigaciones que generan innovación día con día y aún no todas las instituciones llevan el control de su producción. 
Para lograr ser almacenado en un RI se deben cumplir con estándares de calidad en la literatura y desarrollo científico.

Lo anterior nos lleva a cuestionarnos ¿Cuál es la importancia de que un estudiante de posgrado elabore al menos un artículo científico durante su formación? García (2020) responde a esta interrogante y presenta en primer lugar que la revista de investigación científica se diferencia de otros medios de divulgación de la ciencia por el contenido innovador y el proceso riguroso de evaluación para lograr ser publicados. También menciona algunos aspectos positivos de la divulgación entre los cuales dice que a partir de una previa validación se previene a la población de sucesos trascendentes en nuestra vida cotidiana, la publicación de hallazgo ayuda a obtener retroalimentación y pueden ser replicados para mejorar los procesos y resolver problemas, los productos resultantes son presentados a público especializado en el tema y público en general, y la más importante, menciona el autor, es que la población esté informada y desarrolle sus capacidades para mejorar su calidad de vida. Como apoyo de su investigación presenta un análisis de la orientación del Programa Nacional de Posgrados de Calidad (PNPC) que explica los aspectos positivos de la orientación en el ámbito profesional y de investigación explicando cómo el estudiante va desarrollando sus habilidades que benefician de manera profesional, así como mejora el pensamiento crítico lo que resulta en trabajos originales y de calidad que aportan mucho a la sociedad, en este trabajo también se incluyó un manual para la redacción adecuada de un artículo y como conclusiones dan pauta a seguir indagando sobre la baja productividad de los artículos de investigación en este nivel de estudios que es de gran importancia para el impulso de la investigación científica.

Los artículos y demás productos académicos actualmente se encuentran almacenados en plataformas digitales en la red.

Sepúlveda et al. (2019) Realizaron un análisis de once de los repositorios de acceso abierto de instituciones de educación superior en México pertenecientes al OpenDoar el cual funciona como directorio internacional de repositorios, por lo que fue necesario utilizar la metodología Construcción Social de la Tecnología (SCOT) la cual comprende todos los actores que participan en el desarrollo, implementación, gestión y uso de los repositorios. Con base en lo anterior se presentaron como resultados las ventajas de incluir la producción académica en los $\mathrm{RI}$ y se mencionan los siguientes: Se pueden incluir productos académicos que no son publicados en revistas, la búsqueda se realiza en sistemas de general sin que estas sean de tipo académico, existen múltiples copias de acceso desde diferentes ubicaciones, las políticas son de gran ayuda para generalizar las publicaciones de cada institución, en el mismo sentido también es importante mencionar que existen ciertos desafíos qué enfrentar al llevar a cabo la publicación de estos productos y dichos sistemas, entre los que destacan: existe una contrariedad entre las políticas de los RI con las editoriales y revistas por la suscripción, en estos sistemas es muy baja la calidad de los metadatos que se presentan lo que resulta en dificultad de visualización, finalmente concluyen una vez realizada la investigación, en que, la mayoría de los repositorios no cuenta con la diversidad de productos académicos necesaria y en algunos casos solo se limitan a incluir tesis. 
La experiencia del usuario es un dato que los desarrolladores de los RI deben tomar en cuenta para innovar, mejorar y hacer más intuitivos estos sistemas de información para lograr un mayor impacto en la comunidad científica y académica, por este motivo González et al. (2018) presentan una investigación que tiene como fin resaltar las opiniones y necesidades de los usuarios sobre estas plataformas. Para el desarrollo de este trabajo utilizó literatura de trabajos que han aplicado la metodología de Diseño Centrado en el Usuario (DCU) que se apega a la creación de sistemas con base en las necesidades del usuario. Como resultados obtenidos se presenta una guía de mejores prácticas para asegurar la información, ya que actualmente se llevan a cabo actividades poco éticas y plagio con los productos depositados en la red.

Los programas para la gestión de RI juegan un papel esencial en la innovación de estos sistemas, que dependiendo las exigencias del usuario se van mejorando día a día, Eíto-Brun \& Lobón-Márquez (2020) presentaron una actualización de las aplicaciones informáticas más populares específicamente Dspace o Eprints que se encuentran en constante evolución enfocados especialmente a las políticas de acceso abierto. Como resultados se presenta una comparación de los sistemas Fedora, Islandora, Samvera, Archivematica y concluyen que se deben combinar para lograr un mejor resultado $\circ$ un sistema estándar que permita una preservación de calidad utilizando estas aplicaciones de código abierto.

\section{RESULTADOS: ANÁLISIS ESTADÍSTICOS DE EXPLORACIÓN WEB}

En la fase de búsqueda se elaboró una lista de veinte RI explorados, a partir de dicha exploración se presenta en la Figura 4 el análisis de los resultados obtenidos.

\section{Figura 4}

Estado de la presencia de artículos en los RI

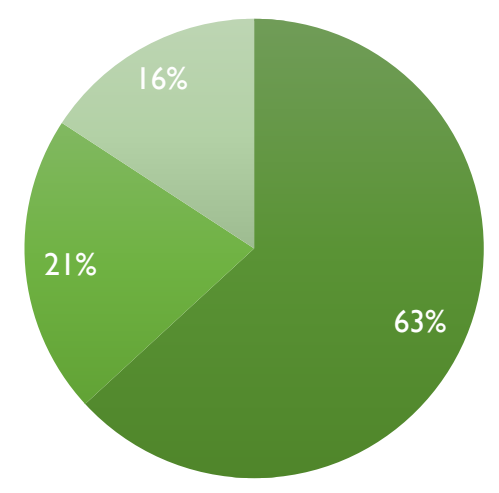

- Clasificados $\quad$ Sin clasificar $\quad$ Solo tesis

En la muestra tomada de RI respecto al estado de los artículos se presentan los siguientes datos: El 63\% de los RI incluyen el apartado artículos que son debidamente clasificados de la siguiente manera:

- Artículos de investigación

- Artículos de divulgación

- Por área académica

- Por áreas de estudio

- Artículos en Redalyc

- Artículos científicos indexados

- Artículos de acceso abierto

- Artículos técnico profesional

- Artículos arbitrados

En cada caso cuentan con el número de trabajos almacenados y sus respectivos filtros de búsqueda. 
En contraste, se observó que el $21 \%$ de los $\mathrm{RI}$ no aplican una clasificación, lo que genera dificultad al realizar la búsqueda de un trabajo en específico. En consecuencia, este tipo de plataformas generan menos impacto entre los usuarios y que esta actividad genere más tiempo en realizarse.

Finalmente, el $16 \%$ se limitan específicamente a solo almacenar tesis, libros, reportes, patentes, prototipos, entre otros, de los distintos niveles de las instituciones.

Es importante presentar la producción actualizada de artículos por institución, hasta septiembre 202 I en cada RI, misma que se muestra en la Figura 5.

\section{Figura 5}

Resultado de la exploración web en los principales repositorios institucionales de México

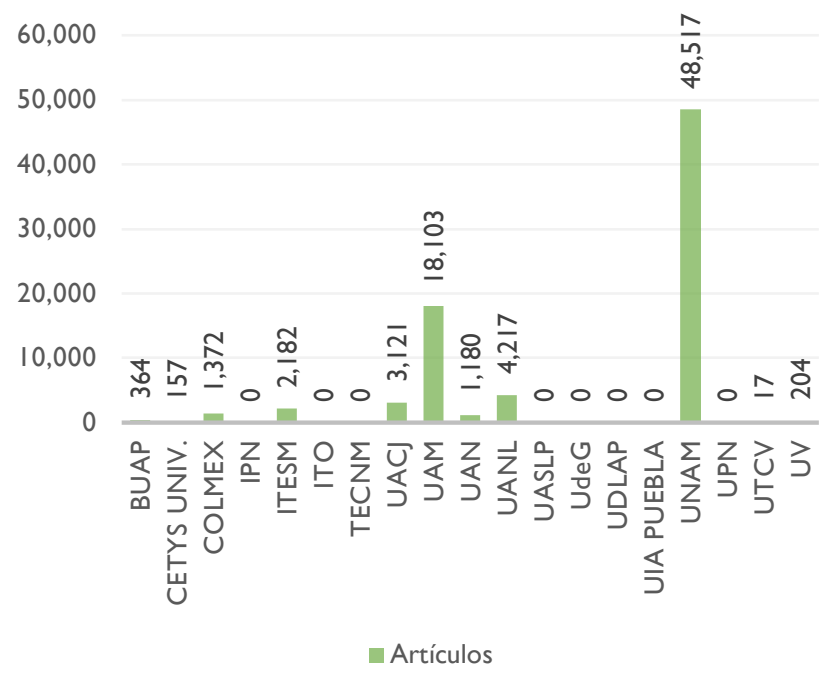

Cada institución se encuentra en distinta etapa de desarrollo de su repositorio institucional por diversas circunstancias, por lo que, las que reflejan cero artículos, se debe a que aún no se realiza el proceso de carga de sus trabajos de investigación que además requieren cumplir protocolos y normas antes mencionados en este trabajo.

\section{CONCLUSIONES}

En conclusión, la producción de artículos crece exponencialmente debido a las investigaciones que realizan las instituciones, y como es debido, es conducente informar a la comunidad científica tomando en cuenta los lineamientos y aspectos legales necesarios, brindando el acceso para apoyar otras investigaciones o recibir retroalimentación de parte de expertos dependiendo el tema, por ello la importancia del OA y los RI.

Como puntos de oportunidad es importante mencionar la necesidad de creación de nuevas herramientas que complementen los sistemas de gestión con la inclusión de las bases de datos de mayor impacto a nivel internacional, el uso de los identificadores de artículos DOI, autores ORCID, Revistas ISSN entre otros estándares internacionales que brinden seguridad de la propiedad intelectual y localización fiable de un trabajo en distintas fuentes y que además sean fáciles de utilizar para el público en general y especializado.

\section{REFERENCIAS}

Barrueco, J. M., y Termens, M. (202I). Digital preservation in institutional repositories: a systematic literature review. Digital Library Perspectives.

Booth, A., Papaioannou, D., y Sutton, A. (2016). Systematic Approaches to a Successful Literature Review (2da ed.). SAGE.

CONACYT. (202I). Repositorio Nacional. https://www.repositorionacionalcti.mx/

Cossio, I., Dieguez, I., y Medina Nogueira, D. (2017). Los repositorios institucionales universitarios. https://www.researchgate.net/publication/321167 67 LOS REPOSITORIOS INSTITUCIONALES UNIVERSITARIOS

Cruz, B., Manuel, J., y Rodríguez, A. (20I7). Guía para la evaluación de repositorios institucionales. https://recolecta.fecyt.es/sites/default/files/conten ido/documentos/2017GuiaEvaluacionRecolectaFE CYT.pdf 
Eíto-Brun, R., y Lobón-Márquez, I. M. (2020). Revisión de programas para la gestión de repositorios digitales: Una actualización. Profesional de la Información, 29(5), I-10, https://doi.org/I0.3145/epi.2020.sep.2I

García, A. G. R. (2020). Estudios de posgrado y elaboración de artículos científicos. I Postgraduate studies and preparation of scientific articles. Utopía y Praxis Latinoamericana, 25, 300315.

González, L., Ramírez-Montoya, M.-S., y GarcíaPeñalvo, F. (20/8). User Experience in Institutional Repositories: A Systematic Literature Review. International Journal of Human Capital and Information Technology Professionals, 9, 70-86.

https://doi.org/10.4018/1]HCITP.2018010105

Ramos Simón, L. F., y Cobo Serrano, S. (2018). El uso de metadatos para la gestión del patrimonio digital mundial en la Sociedad de la Información.

http://ru.iibi.unam.mx/jspui/handle/IIBI_UNAM/C $\underline{\mathrm{L}} \mathrm{s}$

Sepúlveda, G. C. T., Reyes, M. M., y Martín, A. S.

(2019). Repositorios de acceso abierto en las instituciones de educación superior en México, una revisión inicial mediante la metodología SCOT. Información, cultura y sociedad: revista del Instituto de Investigaciones Bibliotecológicas, 40, 6076.

UNESCO. (202I). ¿Qué es acceso abierto? https://es.unesco.org/openaccess/\%C2\%BFqu\%C3\%A9-es-acceso-abierto

Xiao, Y., y Watson, M. (2019). Guidance on conducting a systematic literature review. Journal of Planning Education and Research, 39(I), 93-II 2. 